\title{
Method of Spare Parts Planning for Missile Equipment Based on Support Effectiveness Simulation
}

\author{
Renxi Luo ${ }^{a}{ }^{*}$, Zhiyu Jia ${ }^{b}$ and Chenhui Zeng ${ }^{c}$ \\ China Aero-Polytechnology Establishment, Beijing, China. \\ a, "sallylrx@163.com, bjiazhiyu301@163.com, czengchh@163.com
}

Keywords: Support effectiveness Simulation, initial spare parts utilization rate, spare fill rate.

\begin{abstract}
Method of Spare Parts Planning for Missile Equipment Based on Effectiveness Evaluation mainly studies a reasonable planning method for the spare parts of missile equipment. This method uses the initial spare parts utilization rate / spare fill rate for the missile equipment as the starting point. It completes the evaluation and optimization of the spare parts configuration of the missile equipment based on the Based-on effectiveness evaluation. It achieves maximum spare parts supportability under the constraints of missile operational availability and support the operational readiness during the storage of missiles.
\end{abstract}

\section{Introduction}

The missile weapon system has the characteristics of high complexity, long-term storage and one-time use. The missile is a special product used in one time. After the delivery of the missile, its life cycle is divided into two stages of storage and use according to the mission profile. The stage of "storage" includes storage in the storeroom, maintenance and transportation and other processes. The stage of "use" includes combat readiness and launching. The storage period should be the main part of the whole service life period of the missile. Therefore, the research on the planning method of missile spare parts is mainly to consider the spare parts configuration in the storage stage.

Spare parts are the important material basis of equipment maintenance and support activities. At present, there are dual contradictions in the implementation of the actual equipment spare parts support mission. On the one hand, the number of parts of spare parts is too small. It is not guaranteed when the spare parts are needed for the maintenance of the product. In this way, the operational availability of equipment is reduced and it affects the execution of military training missions and the implementation of the combat readiness mission. On the other hand, spare parts are backlog in large quantities and it causes the dual waste of spare parts resources and storage space. Therefore, in view of the characteristics of missile equipment and its spare parts, the spare parts configuration scheme should be reasonably selected. It is of great significance to realize the precision guarantee of missile equipment spare parts.

\section{Analysis of Influencing Factors on Spare Parts Utilization Rate / Spare Fill Rate}

The configuration of the spare parts of the missile equipment is the direct influence factor of spare parts utilization rate / spare fill rate of the initial spare parts. The storage mission model, product design scheme and maintenance plan of the missile affect the requirements of spare parts, and then it indirectly affects spare parts utilization rate / spare fill rate of the initial spare parts.

The influence factors of the initial spare parts utilization rate / spare fill rate of the missile equipment are shown in the following figure. 


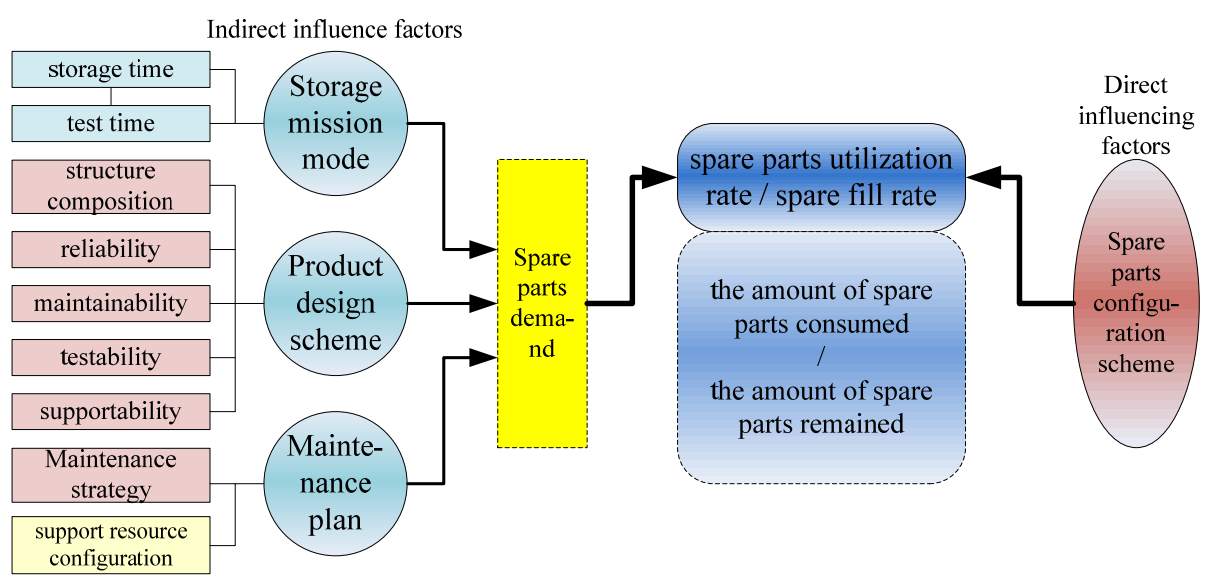

Figure 1. Analysis of influence factors of spare parts utilization rate / spare fill rate

From the figure above, it can be seen that a missile storage mission requirement includes storage mission and detection mission. The mission is the source of the maintenance support activities for the needs of the spare parts. The missile equipment design includes the composition of the product structure and the design level of reliability, maintainability, testability and supportability of the missile. The structure consists of all LRU (line replaceable unit) of the missile equipment. The reliability design level determines the failure probability during the LRU storage. The higher the level of reliability design, the smaller the probability of the failure. The demand for the design of maintenance support is less, and the demand for spare parts will be reduced accordingly. The design level of maintainability, testability and supportability has a direct impact on the types of spare parts needed for maintenance, testing and supporting, as well as the available stock of spare parts. Therefore, these factors also affect the spare parts utilization rate / spare fill rate. The maintenance scheme mainly includes the maintenance strategy after the equipment failure and the spare parts configuration scheme. For maintenance strategies, different strategies for direct maintenance or replacement maintenance based on different types of failure is chosen. Different strategies determine whether there is need for spare parts. For the spare parts configuration scheme, in order to complete a certain mission, the configuration of spare parts including spare parts configuration, determines the ability to repair equipment failure. If the spare parts are adequately configured, the repair is done mainly by replacing the spare parts. If the spare parts are not well configured, it is also necessary to repair the fault by repairing the fault parts. These two different conditions also have different effects on the spare parts demand, thus affecting the spare parts utilization rate / spare fill rate.

Through the analysis of the factors affecting the spare parts demand, it can be seen that in order to effectively realize the analysis and evaluation of the initial spare parts utilization rate / spare fill rate of missile equipment and give a reasonable configuration scheme for the spare parts of the missile equipment, the above factors must be taken into consideration, and the interaction between these factors is taken into consideration and comprehensive measurement.

\section{Overview of Missile Equipment Simulation Model}

The spare parts planning method of missile equipment is mainly based on support effectiveness simulation. It performs related simulation modeling work and evaluates the operational availability of missile and the initial spare parts utilization rate / spare fill rate of equipment.

In this paper, it uses the support effectiveness simulation evaluation system IL Slab-Sims. Based on the discrete event simulation method, it establishes the logical relationship between missile equipment RMS characteristics, storage missions, support resources and maintenance activities. It simulates the activities of missile equipment storage, regular inspection, maintenance and replacement, spare parts supply and other activities. It uses Monte Carlo simulation method, and then calculates the effectiveness of the whole support system.

The simulation model mainly includes a missile equipment product model, a storage mission model, a support resources model and a support process model. Among them, product model, mission 
model and support resource model are static descriptions of all aspects and are input models of overall simulation. The model of the support process is mainly dynamic model. It connects the above static input and depicts the interrelationship between these factors. The model of the support process characterizes the specific simulation process. The modeling of support effectiveness evaluation is the statistics of all indicators in the above simulation process. It obtains the level of each parameter according to the specific evaluation model of each parameter, and it is the output of simulation.

The modeling objects of missile support effectiveness simulation and evaluation include the product platform, storage mission plan, operation and maintenance process of missile equipment, and support resources. Missile equipment and support resources are entities. The operation and maintenance work are a process, and the mission is to drive events in the process of operation and maintenance activities. The overall framework of a missile equipment support effectiveness simulation and evaluation model is shown below.

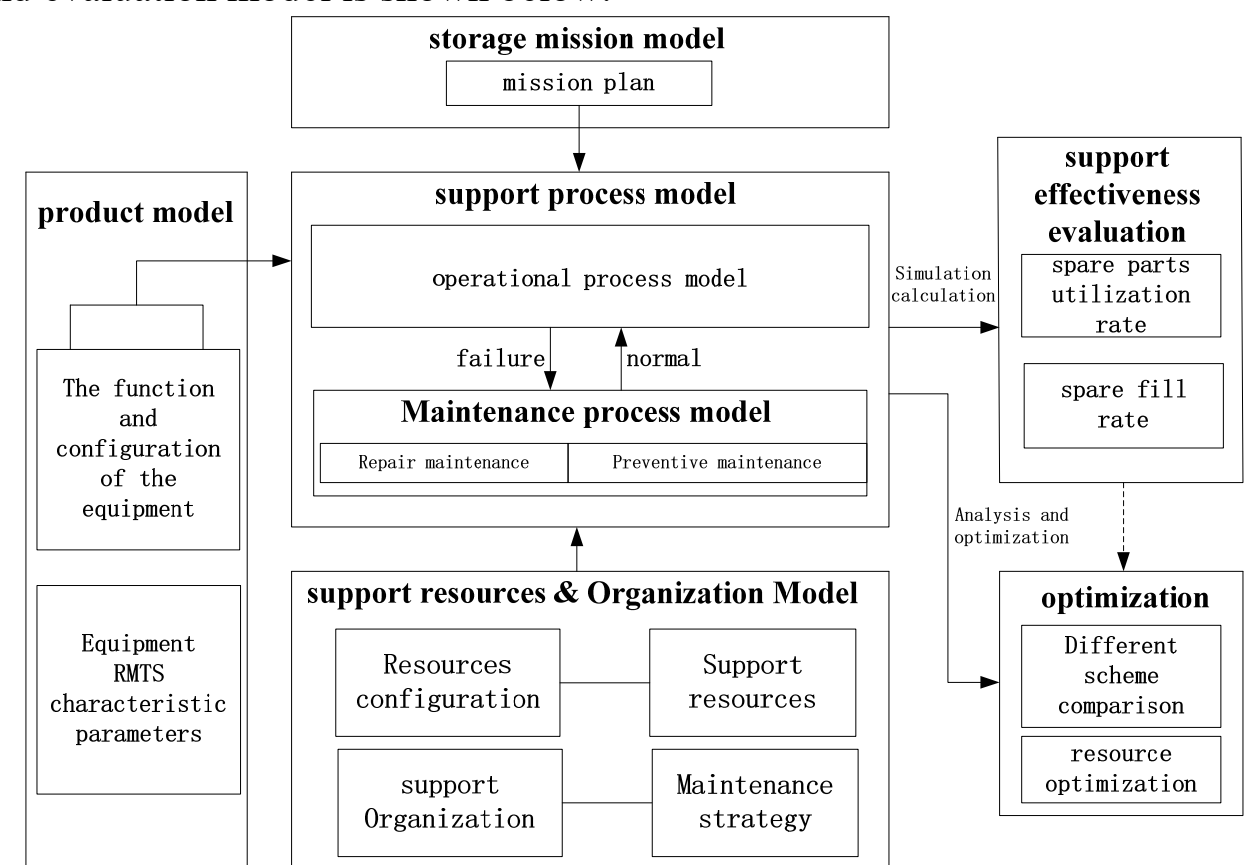

Figure 2. General framework of simulation evaluation model for missile equipment support effectiveness

From the above picture, in order to put forward a reasonable plan for the spare parts of the missile equipment, a comprehensive evaluation of the support effectiveness of the missile is carried out, and the initial spare parts utilization rate / spare fill rate of the missile equipment is studied. First, the missile equipment product model, mission model and support resources model are established, which mainly realizes the static description of the influencing factors of missile support effectiveness. Based on activity network graph modeling method, a support process model is established to complete the dynamic simulation of missile operation and maintenance process and the above static model is organized. Further, according to different parameter evaluation algorithm, it can achieve comprehensive evaluation of missile support effectiveness. The spare parts utilization rate and the spare fill rate of missile equipment are evaluated. Based on the evaluation results, trade-off between different schemes and the optimization of the configuration of maintenance resources are obtained.

\section{Modeling Analysis}

According to the system composition of a typical missile electronic device, it is determined that this electronic device includes 6 LRUs, one A type LRU, one B type LRU, four C type LRUs, and the failure rate of each LRU under storage conditions.

A missile model is established in the simulation evaluation system of the support effectiveness, and each missile is equipped with one of these electronic devices. Suppose that the failure time of electronic equipment is exponentially distributed. According to failure rate, the mean time between 
failures (MTBF) of each LRU can be estimated as input data for electronic equipment failure. The storage of this electronic equipment is not less than 18 years after the delivery of the missile, and it is not maintained during the storage period. The typical mission profile is to carry out the 18 -year storage mission, and a regular testing is carried out every three years. During regular testing, if the device is not defective, it will continue to store. If the equipment breaks down, it replaces the new part and continues storage. At present, missiles at home and abroad usually use the three-level maintenance system including operation-level maintenance, intermediate-level maintenance, and deport-level maintenance. At the operation-level, LRU repair is usually done by replacing part directly. Therefore, LRU is used as a spare part in the support process of a certain type of missile. In view of the long life and high reliability of the missile, the maintenance and replacement time of the missile LRU is usually short relative to the storage time, so the effect of the maintenance and replacement time on the missile LRU can be ignored. In order to prolong the storage life of the missile and ensure the readiness and operational capability of the missile, it is necessary to provide a certain amount of spare parts for the parts that can be replaced in the maintenance activities during the periodic testing. During the regular storage testing of missile, the number of spare parts allocated is mainly considered for support resources. In the storage process, the spare parts may also fail due to the influence of the storage environment factors. Therefore, the number of spare parts as support resources should consider the storage failure. The spare parts configuration of the electronic equipment is fifteen a type spare parts, one B type spare part and one $\mathrm{C}$ type spare part. The simulation results are as follows.

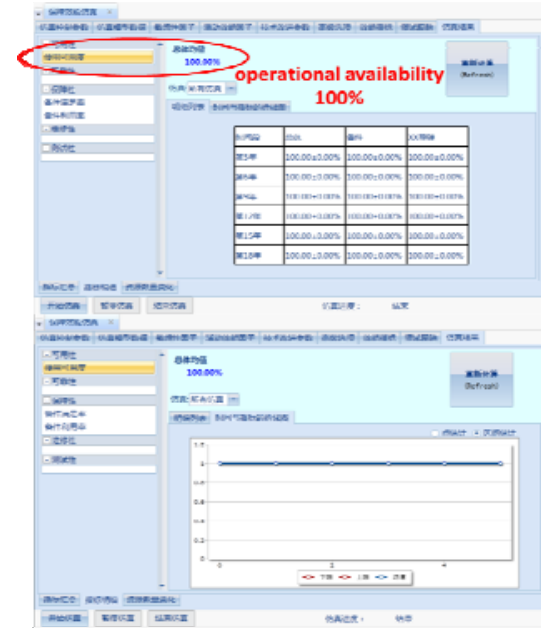

(a)

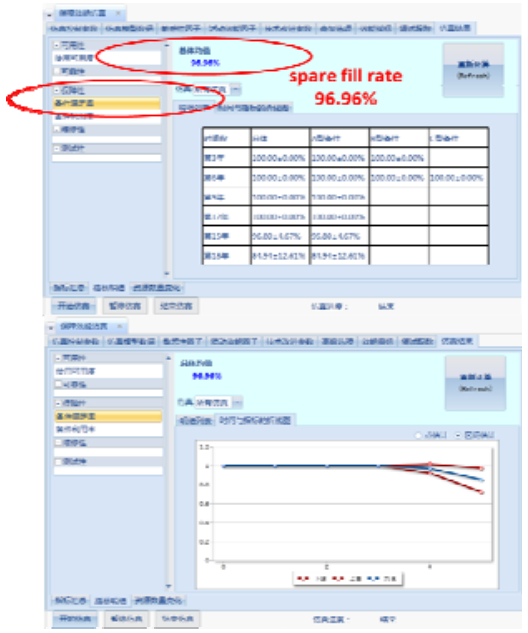

(b)

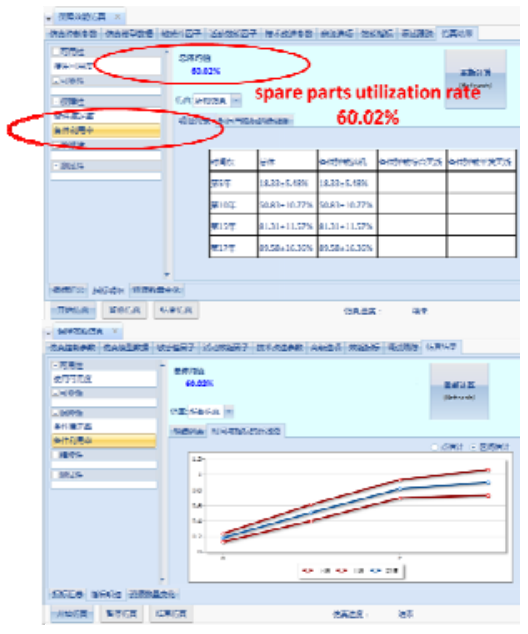

(c)

Figure 3. Simulation results of case analysis

\section{Conclusion}

This paper mainly focuses on the research and application case analysis of spare parts planning for missile equipment based on support effectiveness simulation. Based on the support effectiveness simulation and evaluation system, it takes the system configuration and storage mission profile of a certain type of electronic equipment as the breakthrough point. The system modeling, mission modeling, support process modeling and support resources modeling of this electronic equipment are completed. Through simulation modeling, configuration optimization scheme of spare parts for this electronic equipment of missile is completed. Under the constraint condition of missile operational availability, the maximum spare part support degree is achieved, and the operational readiness of a certain missile during storage is supported. How to comprehensively consider the index constraint relationship of spare parts utilization rate and spare fill rate, it needs further research. Under the constraint of operational availability of missile, it achieves maximum spare part support capability and support the operational readiness of missile in the period of storage. It needs further research that how to comprehensively consider the index constraint relationship of spare parts utilization rate and spare fill rate. 


\section{References}

[1]. Maarten Dresden, Joachim Arts, Geert-Jan van Hutu, Jan Willem Rustenburg, Bob Heisman, "Maintenance spare parts planning and control: a framework for control and agenda for future research Production Planning \& Control. 2015 (5).

[2]. Zhou Yeung Z Zhou Y, "Maintenance support process modeling and simulating technology for aviation materiel 10th International Conference on Reliability, Maintainability Safety. 2014.

[3]. Jian HI Yu, Chen Qian Boa, Zeng Zhao Yang, Xu Zhen Han, Goo Xiao, Jin Xing, "Methods of Maintenance Resources Configuration and Optimization for AMS Based on Effectiveness Evaluation" in Advanced Materials Research. 2012 (476). 